\title{
Islam in the Digital Age: E-Jihad, Online Fatwas and Cyber Environments
}

London, Pluto Press, coll. « Critical Studies on Islam »,2003, 237 p. \& Patrick Haenni, L'islam de marché. L'autre révolution conservatrice Paris, Le Seuil, coll. « La République des idées », 2005, 108 p.

Yves Gonzalez-Quijano

\section{OpenEdition}

Édition électronique

URL : http://journals.openedition.org/assr/3479

DOI : 10.4000/assr.3479

ISSN : $1777-5825$

Éditeur

Éditions de l'EHESS

Édition imprimée

Date de publication : 1 mai 2006

Pagination : 147-299

ISBN : 2-7132-2092-0

ISSN : 0335-5985

Référence électronique

Yves Gonzalez-Quijano, «Islam in the Digital Age: E-Jihad, Online Fatwas and Cyber Environments », Archives de sciences sociales des religions [En ligne], 134 | avril - juin 2006, document 134-16, mis en ligne le 05 septembre 2006, consulté le 21 septembre 2020. URL : http://journals.openedition.org/ assr/3479; DOI : https://doi.org/10.4000/assr.3479

Ce document a été généré automatiquement le 21 septembre 2020.

(c) Archives de sciences sociales des religions 


\section{Islam in the Digital Age: E-Jihad, Online Fatwas and Cyber Environments}

London, Pluto Press, coll. « Critical Studies on Islam »,2003, 237 p. \& Patrick Haenni, L'islam de marché. L'autre révolution conservatrice Paris, Le Seuil, coll. «La République des idées », 2005, 108 p.

Yves Gonzalez-Quijano

1 Entamées par la publication en 2000 de Virtually Islamic. Computer-mediated Communication and Cyber Islamic Environments (Cardiff, University of Wales Press) et prolongées l'année suivante avec celle de The Good Web Guide to World Religions (Londres, The Good Web Guides), les recherches de Gary Bunt, chercheur à l'Université de Wales, ont connu un prolongement récent avec la parution de son troisième ouvrage Islam in the Digital Age: E-Jihad, Online Fatwas and Cyber Islamic Environments (2003). Il travaille aujourd'hui sur le phénomène des blogs dans le monde musulman (pour plus de détails, $c f$. le site www.virtuallyislamic.com).

2 Gary Bunt fait donc partie des (trop rares) chercheurs qui ont choisi, depuis plusieurs années, de chercher à comprendre les effets du développement spectaculaire des techniques de l'information et de la communication dans le domaine du religieux, et plus spécifiquement dans celui des pratiques propres à l'islam. Aujourd'hui, une telle orientation ne surprend plus, ou presque. Mais à l'époque où il a commencé ses recherches, quelques années seulement après la banalisation des usages de la Toile (vers le milieu des années 1990), il fallait, pour se consacrer à un tel sujet, non seulement faire partie de ceux qui percevaient déjà le potentiel des nouvelles technologies, mais également ne pas craindre d'aller à contre-courant de nombre d'idées reçues. Ils étaient nombreux en effet à considérer, spontanément, que les nouveaux outils de la communication mondialisée ne concernaient que les pays les plus industrialisés, ou bien encore à estimer que le monde du religieux, et plus encore dans sa composante musulmane, ne pouvait être que marginalement touché par des innovations limitées à un cercle restreint de spécialistes. Les événements 
internationaux qui ont marqué le début du troisième millénaire, à commencer par les attentats contre le World Trade Center du 11 septembre 2001 (qui sont au cœur de l'ouvrage présenté ici), se sont chargés de modifier la donne et il n'est plus guère d'essais sur l'islam et le monde musulman qui ne consacre, de façon plus ou moins détaillée, quelques pages à " l'Islam du web». Il reste que peu d'analystes peuvent se targuer, comme l'auteur de Islam in the Digital Age, d'avoir mené un travail de fond sur plusieurs années, en associant, ce qui n'est pas la moindre difficulté, l'érudition " classique ", y compris dans sa composante islamologique, à de multiples savoir-faire, plus techniques, capables de rebuter plus d'un spécialiste de sciences sociales.

3 Pour l'essentiel, cet ouvrage s'arrête à deux aspects de l'univers cybernétique musulman (Cyber Islamic Environments) : la question du e-jihad, sous toutes ses formes, et celle de l'autorité religieuse, et de son évolution, sous l'effet de la généralisation des pratiques numériques. Gary Bunt développe l'idée selon laquelle la notion de e-jihad constitue, et singulièrement dans le monde de l'après 11 septembre, la traduction, dans la sphère musulmane de l'internet, des concepts de "guerre cybernétique " (net-war, cyber-war) développés aux États-Unis principalement dès le milieu des années 1990. Exposée dans le deuxième chapitre (The « Digital Sword » and Defining the " e-jihad »), cette thèse est défendue (chap. 3 : Hactivism, Hacking and Craking in the name of Islam) par l'analyse du cyber-activisme islamique (également appelée hacktivism : sur cette notion, cf. http://fr.wikipedia.org/wiki/Hacktivisme), en l'occurrence les différentes formes du « combat pour le jihad » sur la Toile et dans les réseaux informatiques.

4 Les manifestations de ce jihad virtuel sont ensuite étudiées dans le nouveau contexte créé par l'apparition soudaine du phénomène Al-Qaïda sur la scène publique internationale. L'auteur présente le matériel recueilli sur différents sites, actifs ou non depuis l'intérieur du monde musulman. Se côtoient ainsi des communautés de fidèles, ou simplement des partisans, du monde musulman d'Asie (chap.4), des militants palestiniens (chap. 5) ou encore différentes voix désireuses avant tout de se démarquer de l'image de l'islam extrémiste associée au terrorisme mondial (chap. 6).

$5 \quad$ S'il est bien entendu trop tôt pour tirer des conclusions définitives sur des phénomènes en constante évolution, l'auteur note toutefois l'importance croissante d'Internet en tant que vecteur d'information ; un vecteur utilisé, par ailleurs, d'une manière de plus en plus fine et adroite, en fonction des contextes d'utilisation. On voit ainsi se constituer sur la Toile une "nébuleuse jihadiste » - ce que Gary Bunt désigne, pour sa part, sous la formule un peu facile de digital sword (le sabre numérique) - qui est à coup sûr un indicateur important sur les « croyances des musulmans contemporains ». Selon la manière dont ces pratiques virtuelles s'articuleront à la réalité, en fonction notamment des évolutions techniques, des capacités d'accès à internet, et de la dissémination, au-delà des élites, d'une culture informatique, on peut s'attendre, ou non, à ce que, via un jihad devenu " global », l'entrée en force de l'islam sur le Réseau en accélère considérablement les modes de diffusion, comme autrefois l'essor de l'imprimé ou celui des mass-médias radiodiffusés... (« ... a shift in models of Muslim propagation as dramatic as that of the widespread use of print media in the nineteenth century, and as pervasive as broadcasting media in the tweentieth century. Jihad has now truly "gone global" (p. 122)»).

6 La seconde partie de l'ouvrage est consacrée à l'analyse des effets liés à la diffusion des pratiques associées à Internet dans les milieux musulmans sur la "prise de décision » (decision making). Usant de la même méthodologie, principalement basée sur 
l'observation de la Toile, l'auteur montre les implications de la multiplication de sites offrant des consultations juridiques en ligne, des bases de données d'avis d'experts ou d'autorités religieuses, des forums de discussions sur ces questions. Que ce soit dans le monde majoritaire sunnite, au sein du monde musulman ou non (chap. 8 et 9), ou encore dans des milieux plus minoritaires, chiites ou soufis (chap.10), on observe partout les effets, difficilement prédictibles encore, d'une réorganisation en profondeur, au sein des acteurs propres au champ religieux comme dans le domaine des relations qu'entretiennent les fidèles avec l'autorité, et ceux qui sont supposés l'incarner. Les pratiques du cyber-activisme mais également les multiples formes du rapport à l'autorité qui se développent sur le Réseau montrent que le processus de transmission du savoir, $\mathrm{y}$ compris dans la sphère du religieux, est à présent entré dans une phase nouvelle, en partie façonnée par la « matérialité » du support numérique.

L'étude de Gary Bunt offre de très nombreuses informations sur des phénomènes d'autant moins connus qu'ils sont récents et relativement difficiles d'accès, pour toutes sortes de raisons, notamment linguistiques et techniques. S'appuyant sur l'observation patiente de sites, fruit à coup sûr de très longues heures passées à explorer les contrées musulmanes du Réseau des réseaux, ses remarques ouvrent nombre de perspectives, affirmées d'ailleurs avec prudence. On peut regretter cependant que l'ouvrage, préparé dans le climat qui a suivi les événements du World Trade Center, fasse la part un peu trop belle aux formes les plus extrémistes (et peut-être aussi les plus susceptibles de disparaître au gré de l'actualité) du discours islamique sur Internet, en regroupant sous un même regard des manifestations politiquement, sociologiquement - et religieusement - aussi diverses que les communiqués de groupes se revendiquant, authentiquement ou non, de la pensée d'Osama Ben Laden et les tenants d'un e-jihad en Palestine... Des exemples, moins radicaux, sont certes produits, qui permettent de lever en partie le voile sur une utilisation différente, plus piétiste, des médias électroniques. Mais le lecteur sent qu'il leur manque d'être suffisamment spectaculaires pour mériter davantage d'analyses, et ce n'est pas le recours aux catégories traditionnelles de l'islamologie ("grand» et "petit» jihad, islam sunnite majoritaire et courants chiites ou soufis minoritaires...) qui permet d'explorer utilement ces autres voies. Islam in the Digital Age met bien en évidence ce qu'il y a de "digital» dans les pratiques contemporaines de l'islam, mais sans guère avancer de réponses à ce qui relèverait d'un «New Age » de l'islam.

8 Pour cet islam "new age ", pour discerner les traits de ces pratiques religieuses en gestation sous l'effet, entre autres facteurs assurément, de la nouvelle économie et de la révolution des télécommunications, il faut se tourner vers un autre essai, L'islam de marché. L'autre révolution conservatrice. Son auteur, Patrick Haenni, fait également référence, au tout début de son texte, aux événements du 11 septembre, mais c'est évidemment pour en tirer des conclusions très différentes. Bien loin de s'enrôler sous la bannière du "clash des civilisations", il estime, au contraire, que les formes actuelles de l'islam politique s'élaborent en fonction d'une sorte d'idéal de la "politique de la morale», laquelle remplace désormais des revendications plus classiques autour de la question de l'État-nation et contribue à rapprocher ainsi le monde musulman d'un certain modèle américain.

9 En effet, la multiplication des déçus de l'islam politique, conjointement à la montée d'une islamisation qui a pris la place de l'islamisme (sur la différence entre ces deux notions, cf. p. 8 , note 3), ouvre un espace propice au développement d'un «islam de 
marché ». Ce mouvement est certes limité, dans la pratique, à certaines élites mais ses échos dans la société sont bien plus vastes car il touche en cascade, par toutes sortes de moyens, des couches sociales beaucoup plus vastes, en particulier en milieu urbain. Ce "nouvel islam» semble promis à un bel avenir car différents phénomènes s'y conjuguent: la cristallisation d'une religiosité individualiste, de nouveaux jeux d'échange entre le religieux et l'économique (et non plus exclusivement entre le religieux et le politique), l'affirmation, dans le champ religieux encore, d'un nouvel esprit d'entreprise, et enfin une politisation néolibérale de l'islam via la constitution de sociétés civiles vertueuses interagissant avec l'État.

10 Cette thèse, rien moins qu'originale, ne serait-ce qu'au regard de la doxa qui pose un antagonisme fondamental entre le monde musulman et l'Occident, P. Haenni la développe dans les quatre chapitres de ce court essai. Il l'illustre également par de très nombreux exemples révélant aux lecteurs nombre de pratiques contemporaines, voire " post-modernes », qui sont loin de correspondre à la vision traditionnelle, à vrai dire assez compassée, qui règne encore chez de nombreux spécialistes de cette religion. Il s'attarde ainsi sur les productions musicales à destination de la jeunesse, sur les thérapies personnelles « islamisées ", les stratégies vestimentaires allant du foulard au street wear islamique, et bien d'autres formes d'une «culture musulmane " globalisée. Bien entendu, les multiples manifestations de l'univers numérique ne sont pas oubliées et l'auteur s'appuie sur la lecture des sites Internet, des forums de discussion, des messages SMS échangés par la jeunesse sur les téléphones portables, autant de supports médiatiques qu'utilise notamment un jeune prédicateur égyptien, Amr Khaled, qui réapparaît régulièrement dans les pages de cet ouvrage.

11 La démonstration de Patrick Haenni a pour point de départ le constat d'une sorte de contournement de la posture islamiste minée, de l'intérieur, par les démarches postmodernes et sécularisantes des militants et déconstruite, de l'extérieur, par «l'expérience au quotidien des thèmes et emblèmes de l'islamisation» (chap. 1). Dès lors, le marché s'offre comme une sorte d'exutoire aux frustrations politiques, imposant ainsi un " gauchissement »- si l'on ose dire ! - du discours, lequel délaisse les catégories du politique pour se tourner davantage vers celles de l'éthique. «Marchandisé » de mille et une manières, l'islam perd de cette austérité volontiers prônée par l'islam politique. Par cette intégration à l'espace public du marché, l'affirmation islamique tend à se consolider, mais c'est toutefois au risque de s'y dissoudre quelque peu, de se réduire au maniement d'une identité suffisamment cryptée pour échapper au rejet et à l'exclusion dans la société globale. On comprend bien, malgré tout, qu'on ne peut se contenter de stigmatiser un tel phénomène en le réduisant à une simple exploitation mercantile de la religion. Cette nouvelle éthique, cette muslim pride des battants du "nouvel islam », ne passe pas par la revendication politique mais par la performance économique (chap. 2).

12 Au seuil du nouveau millénaire, c'est donc à une sorte de révolution culturelle que l'on assiste, selon P. Haenni. Une forme de "théologie de la prospérité bien musulmane " (p. 64) tend à se substituer à un éthos de l'ascétisme et du fatalisme volontiers cultivé par l'islamisme. Comme le montre toute une série de trajectoires marquantes, celle d'un Mohamed Ahmed al-Rashed, une des figures importantes de la mouvance islamiste dans le monde arabe, mais aussi celle d'un Aa Gym en Indonésie ou d'un Fethullah Gülen en Turquie, une "théologie de la prospérité », porteuse d'une "utopie managériale », tend à se substituer aux précédentes formes de mobilisation (chap. 3). 
Une nouvelle configuration politico-religieuse peut ainsi se constituer, dans laquelle le discours identitaire de l'islam politique, qui demeure articulé sur une conception centrale de l'État-nation, cède la place à un «islam de projets", réticulaire et doté d'une identité malléable, sinon molle. Alors que l'islamisme «traditionnel» prône encore la prise de contrôle des appareils étatiques, le nouvel « islam de marché » vante le rôle d'une sorte de faith based initiative où l'initiative publique doit être déléguée aux opérateurs religieux (chap.4). Bien loin d'incarner cet absolu de l'altérité, cette crispation identitaire lancée comme un défi à la globalisation triomphante, l'« islam de marché » s'inscrit le long d'un «axe de la vertu » fondé sur la religion, la morale, les œuvres et le marché. Il s'oppose, par conséquent, d'autant plus à l'Europe des Lumières, de la raison laïque et étatiste, qu'il est proche, par tous ces aspects, de la « révolution conservatrice » américaine...

Petit par son format, cet essai n'en brasse pas moins nombre d'idées nouvelles, dont on n'a pas fini de débattre, à coup sûr. La démonstration est brillante, les exemples apportés suggestifs, mais l'approche est si novatrice qu'elle ne peut manquer de susciter des interrogations. La thèse principale pour commencer, qui pose une certaine proximité idéologique entre deux révolutions conservatrices, l'étasunienne et l'islamique, risque de provoquer autant de surprise que de commentaires!... Mais bien d'autres questions viennent à l'esprit, notamment sur le bien-fondé d'une démarche qui rassemble sous une même "étiquette ", celle de l'islam de marché, des pratiques aussi diverses que celles que l'on peut observer en Turquie, en Égypte, en Indonésie, au Pakistan ou ailleurs encore, par exemple dans les populations musulmanes émigrées... En procédant de la sorte, ne risque-t-on pas de négliger l'articulation à des contextes tout de même fort différents, en cédant à une certaine forme d'essentialisme auquel, d'ailleurs, l'islam (l'Islam ?) est trop souvent soumis? S'il est à coup sûr bienvenu de rappeler que la globalisation des flux économiques, des réseaux d'informations, des répertoires symboliques n'épargnent pas les sociétés d'islam, faut-il pour autant poser une réponse particulière de cette religion, ou des cultures associées à cette religion ? Faut-il penser que «l'islam n'est pas soluble dans la consommation » pour poser ainsi l'essor d'un "islam de marché »? Ou bien noter, plus simplement, la mutation de pratiques dans un contexte général d'exposition des sociétés (ou de groupes sociaux) naguère « périphériques » à la centralité mondialisatrice?

\section{AUTEUR}

YVES GONZALEZ-QUIJANO

Gary R. Bunt 